\title{
Homogenized finite element models can accurately predict screw pull-out in continuum materials, but not in porous materials
}

\author{
M. Einafshar, A. Hashemi and G.H. van Lenthe
}

\section{Abstract}

Background and Objective: Bone screw fixation can be estimated with several test methods such as insertion torque, pull-out, push-in and bending tests. A basic understanding of the relationship between screw fixation and bone microstructure is still lacking. Computational models can help clarify this relationship. The objective of the paper is to evaluate homogenized finite element (hFE) models of bone screw pull-out.

Methods: Experimental pull-out tests were performed on three materials: two polyurethane (PU) foams having a porous microstructure, and a high density polyethylene (HDPE) which is a continuum material. Forty-five titanium pedicle screws were inserted to 10,20 , and $30 \mathrm{~mm}$ in equally sized blocks of all three materials $\left(\mathrm{N}=5\right.$ /group). Pull-out characteristics i.e. stiffness $(\mathrm{S})$, yield force $\left(\mathrm{F}_{\mathrm{y}}\right)$, peak pull-out force $\left(\mathrm{F}_{\mathrm{ult}}\right)$ and displacement at $\mathrm{F}_{\text {ult }}\left(\mathrm{d}_{\mathrm{ult}}\right)$ were measured. hFE models were created replicating the experiments. The screw was modeled as a rigid body and $5 \mathrm{~mm}$ axial displacement was applied to the head of the screw. Simulations were performed evaluating two different conditions at the bone-screw interface; once in which the screw fitted the pilot hole exactly ("free-stressed") and once in which interface stresses resulting from the insertion process were taken into account ("pre-stressed").

Results: The simulations representing the pre-stressed condition in HDPE matched the experimental data well; $\mathrm{S}, \mathrm{F}_{\mathrm{y}}$, and $\mathrm{F}_{\text {ult }}$ differed less than $11 \%, 2 \%$ and $0.5 \%$ from the experimental data, respectively, whereas $\mathrm{d}_{\mathrm{ult}}$ differed less than $16 \%$. The free-stressed simulations were less accurate, especially stiffness (158\% higher than the pre-stressed condition) and $\mathrm{d}_{\mathrm{ult}}(30 \%$ lower than pre-stressed condition) were affected. The simulations representing PU did not match the experiments well. For the $20 \mathrm{~mm}$ insertion depth, S, $\mathrm{F}_{\mathrm{y}}$ and 
$25 \mathrm{~F}_{\text {ult }}$ differed by more than $104 \%, 89 \%$ and $66 \%$, respectively from the experimental values. Agreement did 26 not improve for 10 and $30 \mathrm{~mm}$ insertion depths.

27 Conclusion: We found that hFE models can accurately quantify screw pull-out in continuum materials such 28 as HDPE, but not in materials with a porous structure, such as PU. Pre-stresses in the bone induced by the 29 insertion process cannot be neglected and need to be included in the hFE simulations.

30 Keywords: Finite element method; Pull-out test; Simulation of screw insertion; Bone analog; Pre-stress

31 modeling; Bone screw

32 Level of evidence: 5 


\section{Introduction}

Bone screws are one of the most commonly used orthopedic implants worldwide. They are used for fixation of complicated bone fractures and for fixation of other implants under complex and cyclic loading [1]. In $2-40 \%$ of patients, these screws dislocate and/or loosen with failure of the surrounding bone as the main reason [2].

Conventional in-vitro testing of the implant-bone structure using cadaveric bones is usually employed to evaluate mechanical fixation of screws $[3,4]$. However, this approach is time-consuming, requires human specimens and is still not well standardized. Moreover, the complications listed above are difficult to predict and accommodate during implant design, leading to limitations in the robustness of each surgical solution [3]. Some studied screw pull-out in-vitro using both synthetic materials [5, 6] as well as human bone [7, 8]. It has been suggested that thread "shape factor" i.e. the average product of pitch and thread depth, is an important factor and that, decreased thread pitch increases screw purchase strength in porous material [6]. The concept of screw pull-out failure is based on the shear failure of an interface between the outer perimeter of the screw and the material in which it is placed. It is assumed that the shear failure of this interface will lead to pull-out in literature, and a thread shape factor is often computed to allow for different thread designs $[5,6,9]$. Novel screw designs are commonly evaluated via static and quasi-static loading according to ASTM F543 [10] using poly-urethane (PU) as bone analog material as specified in ASTM F $1839[11,12]$. It is worth to mention that the real in-vivo loosening of bone screws are affected by dynamic and cyclic loadings which is not implemented in ASTM F543. The PU foam as indicated in the ASTM standard has pore sizes ranging from $0.5 \mathrm{~mm}$ to $2.0 \mathrm{~mm}$. Hence, whereas this standard ensures consistent and uniform material with properties similar to human cancellous bone, it does not necessarily ensure a proper representation of bone microstructure.

In contrast with experimental tests, computer simulations can provide a more efficient screening process for new design ideas or research questions and can provide cost savings as well as a reduced need for valuable tissue samples [13]. Several numerical models have been developed over the last few years aiming 
to predict the deformation that occurs during a pull-out test of a bone screw. Due to the complex microstructure, different simplifying assumptions have been made, such as the use of a cylinder as

61 simplified screw geometry [14], and perfect bonding between the screw and bone [15-18]. Finite element

62 (FE) simulation of bone-screw interface has been carried out using either Micro FE [19] or homogenized

63 FE (hFE) [20].

64 Different levels of complexity can be applied in FE modeling, i.e., with respect to geometry, material 65 properties and interface conditions. The bone anisotropy and microstructural variability between patients complicate the design of screw implants i.e. different screw designs may give different results in different subjects since each piece of trabecular bone is unique. Micro finite element $(\mu \mathrm{FE})$ analysis has a high potential to resolve these phenomena, but still require proper implementation of the underlying non-linear effects and representation of the bone-screw interface [21]. Only a limited number of studies [21] have compared the results of numerical models with mechanical pull-out tests demonstrating a good agreement

71 with either stiffness or strength [11]. Thus far, no data on primary stability of implants has been reported in

72 terms of yield force and displacement at ultimate force, indicating a need for further development in this 73 area.

$74 \mathrm{hFE}$ (also known as continuum FE) models offer an alternative with reasonable computational efforts even 75 for entire bone-implant systems. While not explicitly resolving the complex geometry and mechanical 76 behavior of the bone-screw interface, continuum models have been shown to be able to predict 77 experimentally measured stiffness [4]. Nonlinear material behavior of the bone has been modeled as an 78 elastoplastic material with multiple yield points [22].

79 Implants are usually inserted in the bone through a press-fit procedure where the drill hole is undersized 80 with respect to the implant. The amount of undersizing is critical because too much undersizing will induce 81 excessive press-fit leading to bone damage, which in turn will decrease primary stability and even lead to 82 implant loosening [23-25]. No standardized technique exists to take these effects into account in finite 83 element studies of pull-out process. Some have neglected these [11], while others have presented several 84 modeling techniques to take the press-fit situation into account, e.g., by incorporating a pre-stress 
configuration $[3,13]$, through displacement of the interface boundaries [26], by reducing the bone material

86 properties within at a specific boundary layer around the implant [27], by accounting for damage occurring

87 at the bone-implant interface [28] and by changing the friction coefficients at the bone-screw interface [29,

$8830]$.

89 This study aimed to quantify hFE pull-out characteristics i.e. stiffness $(\mathrm{S})$, yield force $\left(\mathrm{F}_{\mathrm{y}}\right)$, peak pull-out

90 force $\left(\mathrm{F}_{\mathrm{ult}}\right)$ and displacement at peak pull-out force $\left(\mathrm{d}_{\mathrm{ult}}\right)$ of screws in continuum materials as well as in

91 porous materials and relate these to experimental tests. The tests included different bone analogs and screw

92 insertion depths.

93

\section{2. Material and methods}

$95 \quad 2.1$ Experiments

96

\subsubsection{Sample preparation}

97 Two different porous PU foams (Sawbones, Pacific Research Corporation, Vashon, Washington, USA) and

98 a solid high density polyethylene (HDPE) sheet (Direct Plastics Ltd, Sheffield, UK) were cut into $4 * 4 * 6$

$99 \mathrm{~cm}^{3}$ blocks. The dimension of the blocks was chosen according to the dimension of the screws (section

100 2.1.3). These polymers are recommended to use for mechanical testing according to ASTM F543 and

101 ASTM F1717 [10, 31]. High density PU (HDPU) and low density PU (LDPU) can mimic cancellous and

102 osteoporotic cancellous bone, respectively [32]. HDPE can replicate human vertebrae and strongly reduces

103 interspecimen variability [33]. The mechanical properties of the three materials as provided by the 104 manufacturers are summarized in Table 1. 
Table 1. Material properties of the bone analog polymers used for both experimental and finite element models. All Poisson's ratios for low density polyurethane (LDPU) and high density polyurethane (HDPU) are considered 0.3 and no pore size was defined for homogenous high density polyethylene (HDPE) materials.

\begin{tabular}{cccc}
\hline Bone analog & $\begin{array}{c}\text { Density } \\
\left(\mathrm{Kg} / \mathrm{m}^{3}\right)\end{array}$ & $\begin{array}{c}\text { Young's Modulus } \\
(\mathrm{MPa})\end{array}$ & $\begin{array}{c}\text { Pore size } \\
(\mathrm{mm})\end{array}$ \\
LDPU & 160 & 23 & $0.5-2$ \\
HDPU & 320 & 137 & $0.5-1$ \\
HDPE & 947 & 1000 & \\
\hline
\end{tabular}

\subsubsection{Pre-drilled hole}

Fifteen HDPE blocks were pre-drilled to depths of 10, 20, and $30 \mathrm{~mm}$ (N=5/group); the $20 \mathrm{~mm}$ depth is recommended in ASTM F543 [10]. Similarly, the two PU foams were cut into blocks (fifteen per foam) and prepared for three insertion depths i.e. 10, 20 and $30 \mathrm{~mm}$ ( $\mathrm{N}=5$ /group) to demonstrate potential effects of insertion depth. The pilot hole size was considered $5.5 \mathrm{~mm}$ based on the recommendation of the manufacturer. The pilot hole preparation has remarkable effects on results $[34,35]$ and all drilling parameters kept constant during tests.

\subsubsection{Screw insertion}

Titanium conical pedicle screws (Fortex, X.spine cooperation, Cruiser Lane, United States of America) were used in this study. The core and thread profile was conical and cylindrical, respectively. The core diameters were $3.35 \mathrm{~mm}$ and $5.35 \mathrm{~mm}$ at the tip and the head portion, respectively. The pitch was constant throughout the screw length and crest thickness gradually increased from distal to the proximal part of the screw (Fig. 1). The screws were inserted into the pre-drilled hole using a torque meter (LT Lutron, TQ8800, Japan). 


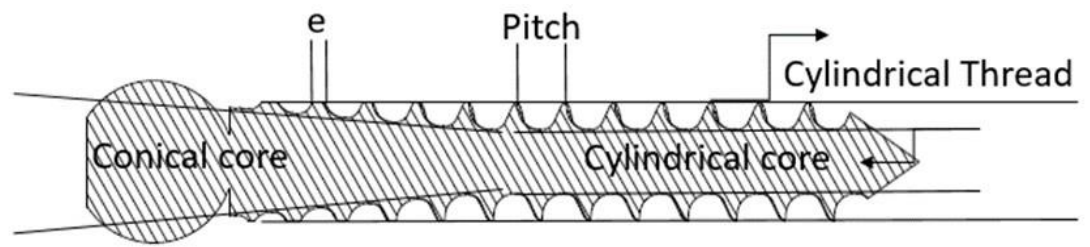

Figure 1. Different features of the pedicle screw used in this study. Crest thickness (e) is the top thickness of a thread.

\subsubsection{Pull-out test}

Pull-out test based on ASTM F543 standard was done using a unidirectional testing apparatus (DTM 25KN, Zwick-Roell, Germany). After placement of the pedicle screw within the HDPE and PU blocks, the orientation of the pedicle screw and tensile hook was set in the coaxial direction and the load cell was set to zero. Displacement control mode with a displacement rate of $5 \mathrm{~mm} / \mathrm{min}$ was carried out for each sample. Load-displacement data were recorded at a rate of $25 \mathrm{~Hz}$. The pull-out force over displacement was recorded for each test case and data acquisition was continued until the screw was pulled out completely. $\mathrm{S}, \mathrm{F}_{\mathrm{y}}, \mathrm{F}_{\text {ult }}$ and $\mathrm{d}_{\text {ult }}$ were calculated for the five samples of the seven groups. $\mathrm{S}$ is the slope of linear elastic part of the force-displacement curve. $\mathrm{F}_{\mathrm{y}}$ was determined as the intersection of a $0.2 \%$ offset line with the force-displacement curve [10].

\subsection{Simulation}

\subsubsection{Geometry and mesh}

Three-dimensional (3D) models of the HDPE and PU blocks and pedicle screw were created using Catia V5R21 and imported in Abaqus CAE 2017 (both software packages by Dassault Systèmes, VélizyVillacoublay, France). Boolean operation was used in order to assemble two parts and create the tapped hole in the 3D block model mimicking the experiments. The screw was finely meshed using 15522 4-node 3D bilinear rigid quadrilateral element. The mass of the screw was 5.1 grams. For the deformable 3D PU and HDPE blocks, 4-node linear tetrahedron element type were used. A radial seeding gradient was performed to obtain better mesh quality. Mesh distortion control was employed to avoid distortional errors. 
152 The mesh of these blocks contained 121082 elements. The mesh convergence analysis was done for 153 different seed sizes of $0.25,0.5$ and $1 \mathrm{~mm} . S$ and $\mathrm{F}_{\mathrm{ult}}$ were evaluated rather than $\mathrm{F}_{\mathrm{y}}$ and $\mathrm{d}_{\mathrm{ult}}($ Fig. 5). The 154 reason that $\mathrm{F}_{\mathrm{y}}$ was not included in the convergency analysis, was the high correlation of Fy to $\mathrm{F}_{\mathrm{ult}}$. Also, $\mathrm{d}_{\mathrm{ult}}$ 155 was excluded because it was hardly affected by mesh size, hence, was not discernable in the convergency 156 analysis. The analyses demonstrated that with a seed size of $0.5 \mathrm{~mm}$ convergency was reached; hence, a $157 \quad 0.5 \mathrm{~mm}$ seed size around the pilot hole was chosen for all subsequent numerical analyses.

\subsubsection{Material properties}

160 The Dynamic Explicit approach was used in this study. An elastoplastic material model with yield strain 161 equal to $10 \%$, and $10 \%$ isotropic hardening $[36,37]$ was implemented for the HDPE blocks. Yield strains 162 equal to 5\%, and 5\% isotropic softening were assigned to the LDPU and HDPU foams [38, 39]. Density 163 and Young's modulus as provided by the manufacturer were assigned (Table 1). Poisson's ratio was set to 1640.3 for all blocks. Due to the notable differences between the elastic properties of the bone analogs and the 165 screw, the latter was considered as a rigid body in all simulations.

\subsubsection{Interface modeling}

167 Surface on surface contact was defined in Abaqus dynamic explicit for the interface between the screw threads and the threaded hole in the bone analogs. Tangential friction contact of 0.6 [37] and hard normal contact were applied between the two bodies.

\subsubsection{Boundary conditions and loading protocols}

171 Simulations were performed evaluating two different conditions at the bone-screw interface. Once in which 172 the screw fitted the pilot hole exactly without causing stress at the bone-screw interface ("free-stressed") 173 and once in which interface stresses resulting from the insertion process were taken into account ("pre174 stressed"). In the latter case, before starting the simulation of the pull-out process, the strains that develop 175 due to the screw insertion process were quantified. Specifically, a radial displacement of $0.5 \mathrm{~mm}$ was 
applied to the threaded part of block (Fig. 2). Two lateral and bottom faces were fixed in pre-straining step

177 in three directions i.e. $\mathrm{U}_{\mathrm{x}}=\mathrm{U}_{\mathrm{y}}=\mathrm{Ur}_{\mathrm{z}}=0$. The radial displacement equals the difference between the outer

178 diameter of the screw and the pilot hole. These 'pre-strains' were transferred to the screw-block model called

179 pre-stressed model in this study. In agreement with the pull-out experimental setup, the top surface of the

180 block was fixed in vertical directions except for a circular section in the center of the top surface of the

181 block replicating experimental setup test, (Fig. 3). All nodes on the screw were coupled to a reference point

182 placed on the head of the screw (Fig. 4). Quasi-static simulations were performed, where variable mass

183 scaling of $10^{-6}$ was used to reduce the analysis time and it was ensured that kinematic energy remained

184 secure i.e. less than $5 \%$ of the internal energy. The simulations were run on Microsoft windows (Intel ${ }^{\circledR}$

185 Xeon ${ }^{\circledR}$ Gold 6152, 96 GB RAM) for an average time of 12 hours per sample. A 5-mm displacement was

applied to this point. The pull-out process was simulated by applying the displacement of the reference

187 point along the longitudinal directional of screw using a one-by-one tabular amplitude in Abaqus. Other

188 displacements and rotational components were set to zero.

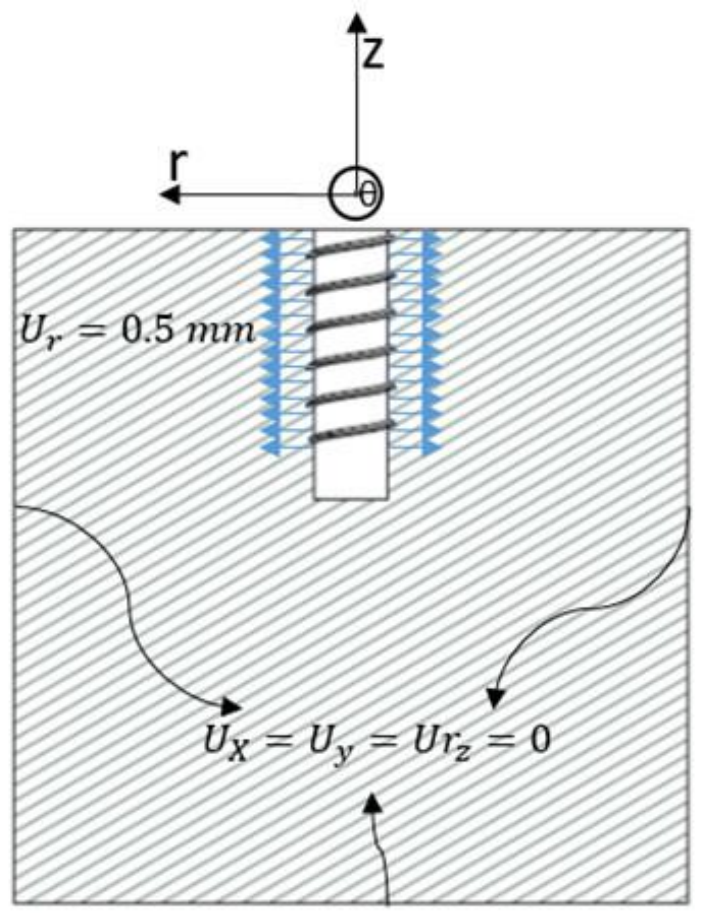



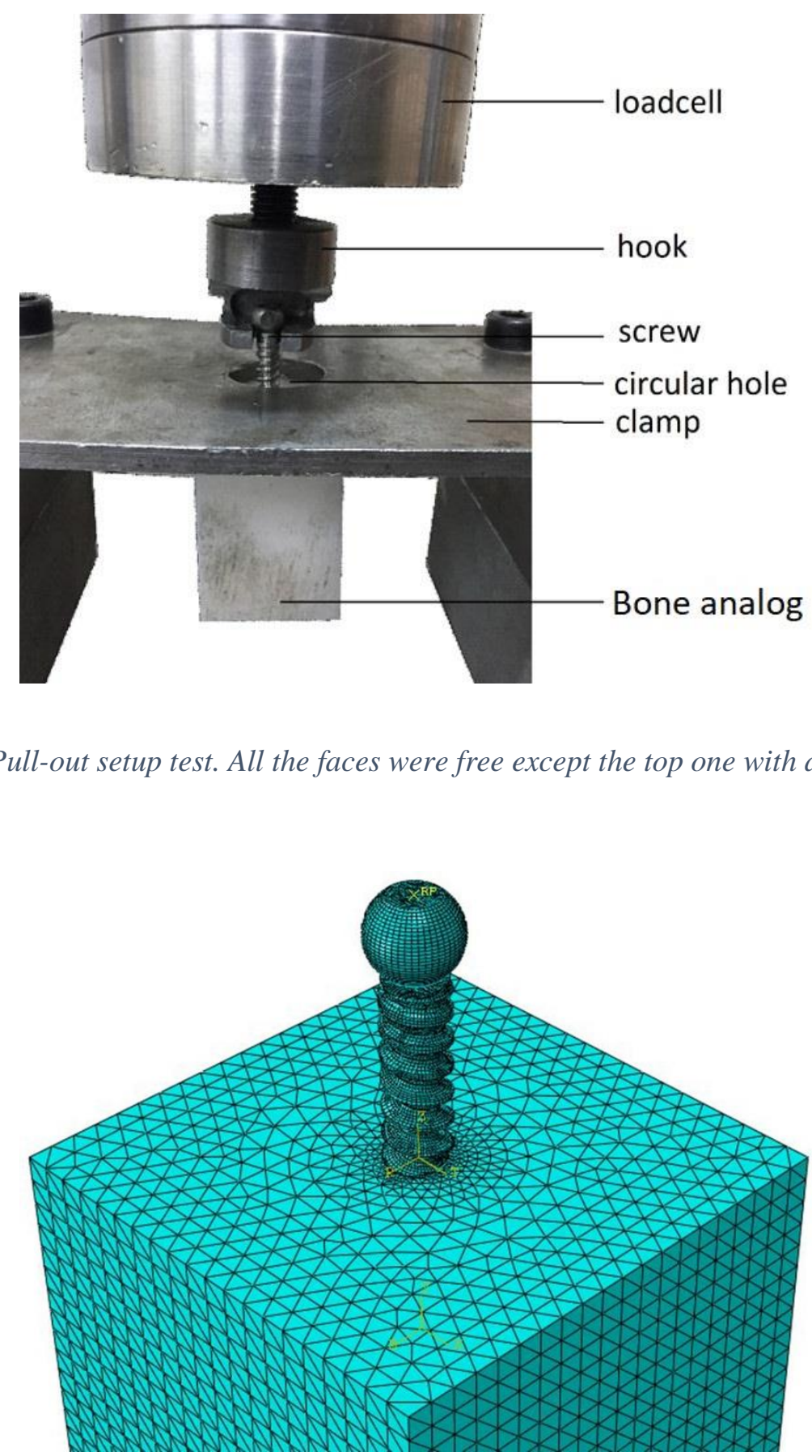

197 Figure 4. The location of reference point and placement of the screw in the test block used to simulate the pull-out process. 
203 Validation of the FE results relative to the experimental measurements was performed for the parameters

$204 \mathrm{~S}, \mathrm{~F}_{\mathrm{y}}, \mathrm{F}_{\mathrm{ult}}$ and $\mathrm{d}_{\mathrm{ult}}$. Every single result was divided by the relevant average experimental result and was expressed as a percentage (Fig. 6). All the data for each test condition, i.e. polymer density and insertion depth were statistically analyzed using one-way ANOVA (Microsoft Excel 2003, Microsoft Corp.,

207 Remond, WA, USA). A p-value of less than 0.05 was considered statistically significant. Furthermore, a 208 Tukey-Kramer honesty significant difference (HSD) post hoc test was used to determine significant 209 differences among the results in each test pair.

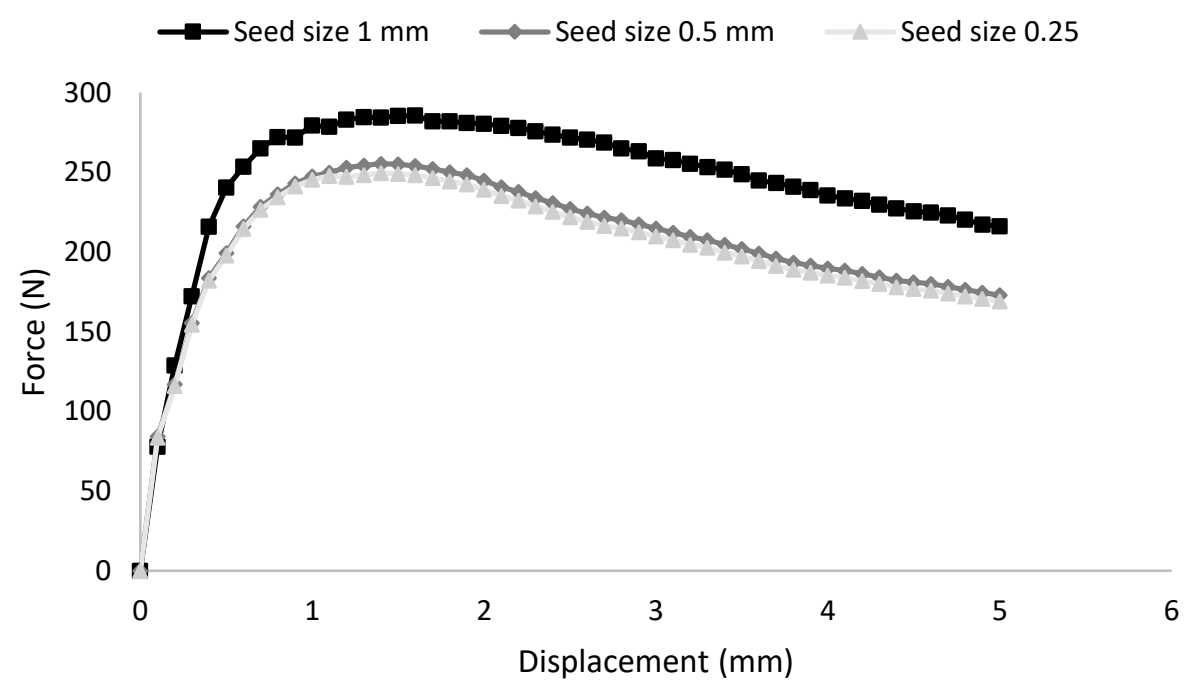




\section{Results}

In all three bone analogs, increasing the insertion depth caused an increase in insertion torque (Table 2).

221 The maximum insertion torque was $218 \pm 2.0 \mathrm{~N} . \mathrm{cm}$ for the $30 \mathrm{~mm}$ insertion depth in HDPE while the 222 minimum one was $17 \pm 1.0 \mathrm{~N} . \mathrm{cm}$ for the $10 \mathrm{~mm}$ insertion depth in LDPU (Table 2).

Table 2. The measured insertion torques for screws inserted into low density polyurethane (LDPU), high density polyurethane (HDPU) and high density polyethylene (HDPE) to three insertion depths.

225

\begin{tabular}{|c|c|c|c|}
\hline \multicolumn{2}{|c|}{ Group } & \multirow{2}{*}{ Insertion Depth (mm) } & \multirow{2}{*}{ Insertion torque (N.cm) } \\
\hline Bone Analog & Density $\left(\mathrm{kg} / \mathrm{m}^{3}\right)$ & & \\
\hline \multirow{3}{*}{ LDPU } & \multirow{3}{*}{160} & 10 & $17 \pm 1.0$ \\
\hline & & 20 & $23 \pm 1.0$ \\
\hline & & 30 & $22 \pm 1.5$ \\
\hline \multirow{3}{*}{ HDPU } & \multirow{3}{*}{320} & 10 & $27 \pm 2.0$ \\
\hline & & 20 & $45 \pm 2.0$ \\
\hline & & 30 & $123 \pm 1.5$ \\
\hline \multirow{3}{*}{ HDPE } & \multirow{3}{*}{947} & 10 & $89 \pm 3.0$ \\
\hline & & 20 & $169 \pm 5.0$ \\
\hline & & 30 & $218 \pm 2.0$ \\
\hline
\end{tabular}

227 In all three materials the $\mathrm{F}_{\mathrm{ult}}$ increased when increasing insertion depths (Table 3). The other pull-out 228 parameters also experienced an increase by increasing the insertion depth from 10 to $30 \mathrm{~mm}$ (Table 3). The 229 PU foam with high density had higher $S, F_{y}$ and $F_{u l t}$ than the low density PU (Table 3). ANOVA test 230 indicated that all four parameters were significantly different between LDPU and HDPU $(\mathrm{p}<0.01)$ and $\mathrm{F}_{\text {ult }}$ 231 in HDPE was higher than in both PU foams (Table 3).

232 In the HDPU foams with the standard $20 \mathrm{~mm}$ insertion depth, the mean $\mathrm{S}, \mathrm{F}_{\mathrm{y}}$ and $\mathrm{F}_{\text {ult }}$ were $375 \%, 228 \%$ 233 and $220 \%$ higher than those in the LDPU (Table 3). S, $F_{y}$ and $d_{u l t}$ values in HDPE are higher than those in 234 PUs (Table 3) and all comparisons between PUs and PE experienced a significant difference $(\mathrm{p}<0.01)$. 
Table 3. Stiffness $(s)$, yield force $\left(F_{y}\right)$, peak pull-out force $\left(F_{u t t}\right)$ and displacement at peak pull-out force $\left(d_{\text {ult }}\right)$ obtained from FE and measured experimentally. The FE analyses were performed twice, one as a free-stressed and one as a pre-stressed for three different insertion depths in a low and high density of polyurethane foam and high density polyethylene.

Group Free-stressed FE

Pre-stressed FE

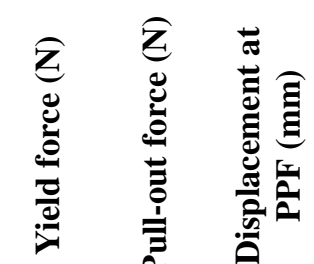

Experiment

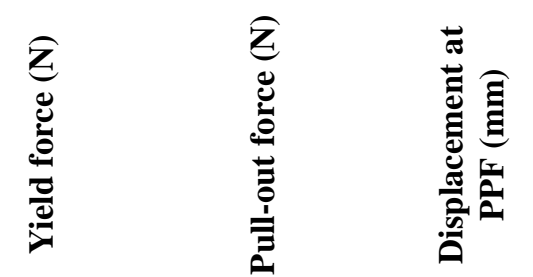

\begin{tabular}{|c|c|c|c|c|c|c|c|c|c|c|c|c|c|c|}
\hline \multirow{3}{*}{ LDPU } & \multirow{3}{*}{160} & 10 & 105 & 79 & 85 & 1.4 & 98 & 69 & 76 & 1.5 & $77.7 \pm 8.3$ & $90.1 \pm 15.1$ & $130.8 \pm 13.3$ & $1.77 \pm 0.21$ \\
\hline & & 20 & 182 & 237 & 252 & 1.6 & 143 & 201 & 223 & 1.6 & $85.8 \pm 12.6$ & $140.6 \pm 16.0$ & $173 \pm 20.4$ & $2.07 \pm 0.65$ \\
\hline & & 30 & 315 & 403 & 422 & 1.9 & 276 & 391 & 402 & 1.6 & $220.4 \pm 56.9$ & $164 \pm 34.7$ & $201 \pm 29.6$ & $3.06 \pm 0.43$ \\
\hline \multirow{3}{*}{ HDPU } & \multirow{3}{*}{320} & 10 & 410 & 379 & 506 & 1.4 & 401 & 315 & 418 & 1.5 & $134 \pm 14.0$ & $159.8 \pm 40.6$ & $195 \pm 30.3$ & $1.62 \pm 0.23$ \\
\hline & & 20 & 1002 & 1169 & 1323 & 1.4 & 988 & 1090 & 1120 & 1.6 & $408.2 \pm 17.9$ & $461.2 \pm 70.6$ & $554 \pm 93.6$ & $2.00 \pm 0.13$ \\
\hline & & 30 & 1576 & 2112 & 2710 & 1.9 & 1395 & 1524 & 2305 & 1.7 & $867.2 \pm 74.7$ & $865.6 \pm 40.4$ & $938.8 \pm 54.2$ & $3.44 \pm 0.27$ \\
\hline \multirow{3}{*}{ HDPE } & \multirow{3}{*}{947} & 10 & 1592 & 1813 & 1927 & 1.3 & 921 & 1602 & 1759 & 2.1 & $845 \pm 34.5$ & $1581 \pm 5.0$ & $1682 \pm 106.0$ & $2.76 \pm 0.11$ \\
\hline & & 20 & 5051 & 4921 & 5249 & 1.8 & 2086 & 4659 & 4824 & 2.6 & $1876 \pm 215.0$ & $4733 \pm 120.7$ & $4799 \pm 174.0$ & $3.10 \pm 0.08$ \\
\hline & & 30 & 6040 & 6918 & 7841 & 1.8 & 3042 & 6348 & 7122 & 2.7 & $2221 \pm 255.0$ & $6112 \pm 222.0$ & $7001 \pm 301.0$ & $3.23 \pm 0.23$ \\
\hline
\end{tabular}


240 In the simulations of the PU foams and HDPE, both the free-stressed and pre-stressed FE models 241 demonstrated that $F_{u l t}$ increased when increasing insertion depth (Table 3). In PUs the increase in $F_{\text {ult }}$ 242 resembled the experimental results, yet, only in a qualitative sense; the absolute values did not match, 243 neither in the free-stressed nor in the pre-stressed conditions (Fig. 6.g and h). More specifically, the results 244 of the free-stressed models of $20 \mathrm{~mm}$ insertion depth were, on average, $128 \%, 110 \%$, and $92 \%$ higher for 245 experimental S, Fy (Fig. 6.a, b, d and e) and $\mathrm{F}_{\text {ult }}$ (Fig. 6.g and h), respectively. Likewise, the results of the 246 pre-stressed model were 104\%, 89\% and 66\% higher for experimental S, Fy (Fig. 6.a, b, d and e) and $\mathrm{F}_{\mathrm{ult}}$ 247 (Fig. 6.g and h), respectively.

248 In the simulations of the HDPE blocks, the results of the pre-stressed model closely matched the 249 experimental findings; specifically, the findings for S, F (Fig. 6.c and f) and $\mathrm{F}_{\mathrm{ult}}$ (Fig. 6.i) were 11\%, 2\%, 250 and $0.5 \%$ higher than the experimentally measured data, while $\mathrm{d}_{\text {ult }}$ (Fig. 6.1) was $16 \%$ lower in $20 \mathrm{~mm}$ 251 insertion depth. In contrast, the results of the free-stressed model in $20 \mathrm{~mm}$ insertion depth deviated much 252 more, especially for $\mathrm{S}$ and $\mathrm{d}_{\mathrm{ult}}$ which were overestimated by $169 \%$ and underestimated by $42 \%$, respectively 253 (Fig. 6.c and 1). Similarly, the predictions of FE pre-stressed models in 10 and $30 \mathrm{~mm}$ insertion depths 254 reveals $9 \%$ and $5 \%$ difference between $\mathrm{FE}$ and experiments for $\mathrm{S}$ and $\mathrm{F}_{\mathrm{ult}}$, respectively while, these 255 percentages are $88 \%$ and 15\% difference for the free-stressed models (Fig. 6.c and i). 

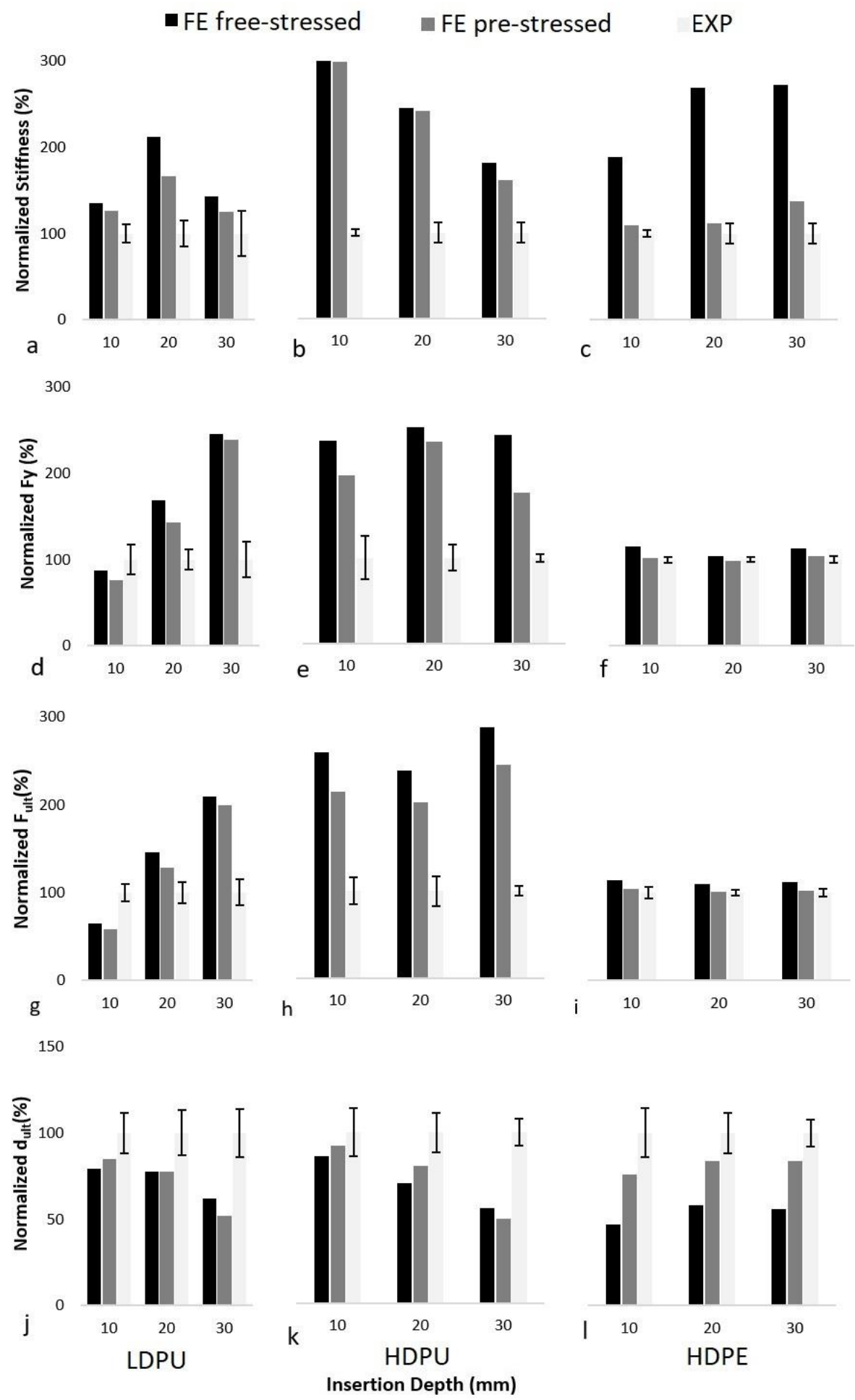

Figure 6. A comparison between the experimentally measured data (the average set to 100\%) and the data 258 as determined from the finite element analyses of the free-stressed and pre-stressed interface conditions for $259 a, b$ and $c)$ stiffness $(S), d$, e and $f$ ) yield force $\left(F_{y}\right), g, h$ and $\left.i\right)$ peak pull-out force $\left(F_{u l t}\right)$ and $j, k$ and $\left.l\right)$ 260 displacement at peak pull-out force $\left(d_{u l t}\right)$ for the $a, d, g$ and $\left.j\right)$ low-density polyurethane (LDPU), $b, e, h$ and 261 k) high-density polyurethane (HDPU) and c, $f$, i and l) high-density polyethylene (HDPE), respectively. For 262 each insertion depth, the average experimental data was set to $100 \%$. 


\section{Discussion}

264 Several studies have experimentally quantified the pull-out characteristics of bone screws using synthetic 265 bone [5, 40, 41], animal samples [42-44] and human cadavers [7, 8, 19]. Furthermore, various studies have 266 numerically evaluated $\mathrm{S}[19,21,45,46]$ and $\mathrm{F}_{\text {ult }}$ using a variety of assumptions among which bonded 267 interfaces, smoothed screw geometry [13] and linear material properties. In this study hFE was used to mimic experimental pull-out test in a LDPU and HDPU foam as well as in HDPE which can be considered

269 a continuum material. In order to simulate the mechanical consequences of the insertion process, two $270 \quad$ labelled modeling approaches i.e. "free-stressed" and "pre-stressed" were compared.

271 We demonstrated that the FE models can replicate well the pull-out characteristics in the PE material, but

272 that results for the porous PU foams were far off. Hence, whereas the material properties of PU as used in 273 this study describe well the mechanical characteristics at the apparent level, they do not represent the 274 mechanical characteristics of the PU material in close vicinity to the screw. This can be explained by the 275 microstructure of the PU and HDPE which differ strongly. The PU foams used in this study consisted of at 276 least $0.5 \mathrm{~mm}$ pores which can be assumed as a porous model while the HDPE did not include any pores at 277 this length scale and can be considered as continuum material. The improved FE pull-out predictions as 278 seen in PE are not a consequence of increased material properties such as density, because the results for 279 the HDPU foams are worse than those for the LDPU foams (Table 3). We hypothesize that in order to 280 replicate the pull-out characteristics in foams a more accurate description of the porous nature of the 281 material in vicinity of the screw needs to be taken into account, which can be achieved using so-called 282 micro-finite element analyses.

283 We found that the radial displacements applied in the pre-stressed models could mimic the insertion process 284 in HDPE. These displacements improved the pull-out characteristics slightly in PU, though in absolute 285 numbers the findings were still far off from the experimentally measured data; this disagreement seems to 286 be dominated by the continuum approach in the hFE models, which neglects the microstructure of the 287 materials. 
In the FE simulations, all pull-out characteristics except $d_{\text {ult }}$ were found to be lower in the pre-stressed model than in the free-stressed one (Fig. 6.a to i). During screw insertion, the threads induce damage to the materials. The damage has not been modeled directly in this study, but the induced stresses in the region of block-screw interface called pre-stresses were considered. Pre-stresses weaken the material especially in the region of interface $[27,37]$. In the FE screw pull-out simulations, material adjacent to the block-screw interface experienced yielding which have already yielded in insertion step.

294 For the standard insertion depth, the $\mathrm{d}_{\text {ult }}$ was on average $2.07 \pm 0.65$ and $2.00 \pm 0.13 \mathrm{~mm}$ for LDPU and 295 HDPU, respectively. The $\mathrm{d}_{\mathrm{ult}}$ for HDPE was on average $3.10 \pm 0.08 \mathrm{~mm}$, i.e. $55 \%$ and $50 \%$ higher than in 296 LDPU and HDPU, respectively (Fig. 6.j, $\mathrm{k}$ and l). These differences are $36 \%$ and $41 \%$ for LDPU and HDPU 297 in $10 \mathrm{~mm}$ insertion depth and 7\% and 5\% in $30 \mathrm{~mm}$ insertion depth in comparison with HDPE, respectively. 298 This difference can be explained by the damage properties of the materials. The higher the fracture 299 toughness, the more the material will deform before rupture. Indeed, the fracture toughness of PU is 47 $\mathrm{J} / \mathrm{m}^{2}$, which is much less than the fracture toughness of HDPE which is $4660 \mathrm{~J} / \mathrm{m}^{2}$ [47]. An explicit solver has been used in this study. Due to the limited convergence, implicit solvers cannot handle the excessive element distortion resulting from the implementation press-fit especially in $\mu \mathrm{FE}$ models [37]. The high deformation related to the implantation press-fit can best be captured by explicit solvers, as they provide the option of element deletion and distortion controlling for highly distorted elements. The explicit hFEM has been able to simulate the implant insertion in isotropic trabecular bone while neglecting the effect of bone geometry and volume fraction [40].

307 In our experimental mechanical tests of the standard $20 \mathrm{~mm}$ insertion depth in PU foams, the $\mathrm{S}, \mathrm{F}_{\mathrm{y}}$ and $\mathrm{F}_{\mathrm{ult}}$ 308 were $375 \%, 228 \%$ and $220 \%$ higher in the high density case as compared to the low density case; this is 309 related to the better grip [32,33]. Moreover, these pull-out characteristics experienced an increase of $183 \%$, $31082 \%$ and $53 \%$ in LDPU and $547 \%, 441 \%$, and $381 \%$ in HDPU, respectively by increasing the insertion 311 depth from 10 to $30 \mathrm{~mm}$ which is in agreement with the study of Vargese et.al [48].

312 There are a few limitations of the current study. First, the insertion process has not been simulated directly 313 but the induced stresses were considered as "pre-stresses" modeled due to calculation cost savings. The 
concept to measure pre-stresses is still remaining inaccurate as Meyer et. al proposed in their study to measure pre-stresses directly by splitting the screw-block into two pieces [49]. Our implementation of applying radial displacement led to the development of pre-stresses adjacent to the threads and equal to the

317 yield stresses of the materials. Second, the post-yield behavior of the PU foams and HDPE has been 318 modelled as a linear softening and linear hardening, respectively. This modeling approach may present a 319 simplification of the physical reality, especially for large strains. For the purpose of this paper this approach 320 is justified because the aim of our study was not to determine the rupture point of the material. And third, $321 \mu \mathrm{FE}$ could be implemented for PU foams but currently the ability of solvers to provide nonlinear contact 322 deformation is limited.

\section{Conclusion}

325 We conclude that the hFE models replicated the pull-out characteristics well in a continuum material, i.e. 326 HDPE, but not in porous materials, i.e., LDPU and HDPU. Furthermore, the implementation of radial 327 displacements to the bone analog improved the prediction of all pull-out characteristics. These radial 328 displacements developed pre-stresses in the model simulating the effects of the insertion process.

Mohammadjavad Einafshar: Methodology, Software, Writing- Original draft preparation, Validation.

Ata Hashemi.: Conceptualization, Supervision, Writing- Reviewing and Editing,

Harry van Lenthe: Conceptualization, Supervision, Writing- Reviewing and Editing,

\section{Acknowledgment}


1. Karami KJ, Buckenmeyer LE, Kiapour AM, Kelkar PS, Goel VK, Demetropoulos CK, Soo TM (2015) Biomechanical evaluation of the pedicle screw insertion depth effect on screw stability under

17. Wirth AJ, Müller R, van Lenthe GH (2012) Augmentation of peri-implant bone improves implant

2. Norris R, Bhattacharjee D , Parker MJ (2012) Occurrence of secondary fracture around intramedullary nails used for trochanteric hip fractures: a systematic review of 13,568 patients. Injury.43:706-11.

3. Varga P, Grünwald L, Inzana JA , Windolf M (2017) Fatigue failure of plated osteoporotic proximal humerus fractures is predicted by the strain around the proximal screws. Journal of the mechanical behavior of biomedical materials.75:68-74.

4. Katthagen JC, Schwarze M, Warnhoff M, Voigt C, Hurschler C, Lill H (2016) Influence of plate material and screw design on stiffness and ultimate load of locked plating in osteoporotic proximal humeral fractures. Injury.47:617-24.

5. Tsai W-C, Chen P-Q, Lu T-W, Wu S-S, Shih K-S, Lin S-C (2009) Comparison and prediction of pullout strength of conical and cylindrical pedicle screws within synthetic bone. BMC musculoskeletal disorders.10:44.

6. Chapman J, Harrington R, Lee K, Anderson P, Tencer A, Kowalski D (1996) Factors affecting the pullout strength of cancellous bone screws. Journal of biomechanical engineering.118:391-8.

7. Zhang QH, Tan SH , Chou SM (2006) Effects of bone materials on the screw pull-out strength in human spine. Medical engineering physics.28:795-801.

8. Feerick EM , McGarry JP (2012) Cortical bone failure mechanisms during screw pullout. Journal of biomechanics.45:1666-72.

9. Bennani Kamane $\mathrm{P}$, Finite element modelling of screw fixation in augmented and non-augmented cancellous bone. 2012, Brunel University School of Engineering and Design PhD Theses.

10. ASTM F543 (2007), Standard specification and test methods for metallic medical bone screws. ASTM International West Conshohocken, PA.

11. Joffre $T$, Isaksson P, Procter P, Persson C (2017) Trabecular deformations during screw pull-out: a micro-CT study of lapine bone. Biomechanics modeling in mechanobiology.16:1349-59.

12. ASTM F1839 (2001), Standard specification for rigid polyurethane foam for use as a standard material for testing orthopaedic devices and instruments. ASTM International West Conshohocken, PA.

13. Inzana JA, Varga P, Windolf M (2016) Implicit modeling of screw threads for efficient finite element analysis of complex bone-implant systems. Journal of biomechanics.49:1836-44.

14. Ruffoni D, Müller R, van Lenthe GH (2012) Mechanisms of reduced implant stability in osteoporotic bone. Biomechanics Modeling in Mechanobiology.11:313-23.

15. Kennedy J, Molony D, Burke NG, FitzPatrick D, Mullett H (2013) Effect of calcium triphosphate cement on proximal humeral fracture osteosynthesis: a cadaveric biomechanical study. Journal of Orthopaedic Surgery.21:173-7.

16. Dubov A, Kim S, Shah S, Schemitsch E, Zdero R, Bougherara H (2011) The biomechanics of plate repair of periprosthetic femur fractures near the tip of a total hip implant: the effect of cablescrew position. Proceedings of the Institution of Mechanical Engineers, Part H: Journal of Engineering in Medicine.225:857-65. stability: Quantification using simulated bone loss. Journal of Orthopaedic Research.30:178-84. 
18. Rungsiyakull C, Chen J, Rungsiyakull P, Li W, Swain M , Li Q (2015) Bone's responses to different designs of implant-supported fixed partial dentures. Biomechanics modeling in mechanobiology.14:403-11.

19. Steiner JA, Christen P, Affentranger R, Ferguson SJ, van Lenthe GH (2017) A novel in silico method to quantify primary stability of screws in trabecular bone. Journal of Orthopaedic Research.35:2415-24.

20. Ovesy M, Voumard B , Zysset P (2018) A nonlinear homogenized finite element analysis of the primary stability of the bone-implant interface. Biomechanics modeling in mechanobiology.17:1471-80.

21. Steiner JA, Ferguson SJ, van Lenthe GH (2015) Computational analysis of primary implant stability in trabecular bone. Journal of biomechanics.48:807-15.

22. Hosseini HS, Clouthier AL, Zysset PK (2014) Experimental validation of finite element analysis of human vertebral collapse under large compressive strains. Journal of biomechanical engineering.136:041006.

23. Gausepohl T, Möhring R, Pennig D, Koebke J (2001) Fine thread versus coarse thread: a comparison of the maximum holding power. Injury.32:1-7.

24. Yadav S, Upadhyay M, Liu S, Roberts E, Neace WP, Nanda R (2012) Microdamage of the cortical bone during mini-implant insertion with self-drilling and self-tapping techniques: a randomized controlled trial. American Journal of Orthodontics Dentofacial Orthopedics.141:538-46.

25. Wang L, Ye T, Deng L, Shao J, Qi J, Zhou Q, Wei L, Qiu S (2014) Repair of microdamage in osteonal cortical bone adjacent to bone screw. PLoS One.9:e89343.

26. Berahmani S, Janssen D, Verdonschot N (2017) Experimental and computational analysis of micromotions of an uncemented femoral knee implant using elastic and plastic bone material models. Journal of biomechanics.61:137-43.

27. Steiner JA, Ferguson SJ, van Lenthe GH (2016) Screw insertion in trabecular bone causes periimplant bone damage. Medical engineering physics.38:417-22.

28. Ovesy M, Aeschlimann M , Zysset PK (2020) Explicit Finite Element Analysis Can Predict the Mechanical Response of Conical Implant Press-Fit in Homogenized Trabecular Bone. Journal of Biomechanics. 109844.

29. Karunratanakul K, Kerckhofs G, Lammens J, Vanlauwe J, Schrooten J , Van Oosterwyck H (2013) Validation of a finite element model of a unilateral external fixator in a rabbit tibia defect model. Medical engineering physics.35:1037-43.

30. MacLeod AR, Pankaj P, Simpson AHR (2012) Does screw-bone interface modelling matter in finite element analyses? Journal of biomechanics.45:1712-6.

31. ASTM F1717 (2014), Standard test methods for spinal implant constructs in a vertebrectomy model. ASTM International West Conshohocken, PA.

32. Hashemi A, Bednar D, Ziada S (2009) Pullout strength of pedicle screws augmented with particulate calcium phosphate: an experimental study. The spine journal.9:404-10.

33. Chao C-K, Hsu C-C, Wang J-L, Lin J (2008) Increasing bending strength and pullout strength in conical pedicle screws: biomechanical tests and finite element analyses. Clinical Spine Surgery. 21:130-8.

34. Ein-Afshar MJ, Rouhi G, Aghighi M , Mortazavi SJ (2016) Alteration of the Thrust Force Versus Number of Drill Bit Usage in Cortical Bone Drilling. Journal of Orthopedic Spine Trauma.

35. Ein-Afshar MJ, Shahrezaee M, Shahrezaee MH, Sharifzadeh SR (2020) Biomechanical Evaluation of Temperature Rising and Applied Force in Controlled Cortical Bone Drilling: an Animal in Vitro Study. J Archives of Bone Joint Surgery.8:605. 
36. Werner B, Ovesy M , Zysset PK (2019) An explicit micro-FE approach to investigate the post-yield behaviour of trabecular bone under large deformations. International journal for numerical methods in biomedical engineering.35:e3188.

37. Ovesy M, Indermaur M , Zysset PK (2019) Prediction of insertion torque and stiffness of a dental implant in bovine trabecular bone using explicit micro-finite element analysis. Journal of the mechanical behavior of biomedical materials.98:301-10.

38. McIntyre A, Anderton G (1979) Fracture properties of a rigid polyurethane foam over a range of densities. J Polymer.20:247-53.

39. Thompson MS, McCarthy ID, Lidgren L, Ryd L (2003) Compressive and shear properties of commercially available polyurethane foams. Journal of biomechanical engineering.125:732-4.

40. Ketata H, Affes F, Kharrat M , Dammak M (2019) A comparative study of tapped and untapped pilot holes for bicortical orthopedic screws-3D finite element analysis with an experimental test. Biomedical Engineering/Biomedizinische Technik.64:563-70.

41. Chatzistergos PE, Magnissalis EA, Kourkoulis SK (2010) A parametric study of cylindrical pedicle screw design implications on the pullout performance using an experimentally validated finiteelement model. Medical engineering physics.32:145-54.

42. Inceoglu S, Ferrara L, McLain RF (2004) Pedicle screw fixation strength: pullout versus insertional torque. The spine journal.4:513-8.

43. Aparicio C, Padrós A, Gil F-J (2011) In vivo evaluation of micro-rough and bioactive titanium dental implants using histometry and pull-out tests. Journal of the mechanical behavior of biomedical materials.4:1672-82.

44. Kettenberger U, Latypova A, Terrier A, Pioletti DP (2015) Time course of bone screw fixation following a local delivery of Zoledronate in a rat femoral model-a micro-finite element analysis. journal of the mechanical behavior of biomedical materials.45:22-31.

45. Varghese V, Ramu P, Krishnan V, Kumar GS (2016) Pull out strength calculator for pedicle screws using a surrogate ensemble approach. J Computer methods programs in biomedicine.137:11-22.

46. Shih K-S, Hou S-M , Lin S-C (2017) Theoretical prediction of pullout strengths for dental and orthopaedic screws with conical profile and buttress threads. J Computer Methods Programs in Biomedicine.152:159-64.

47. Tjong S , Bao S (2007) Fracture toughness of high density polyethylene/SEBS-g$\mathrm{MA} /$ montmorillonite nanocomposites. Composites science technology.67:314-23.

48. Varghese V, Kumar GS, Krishnan V (2017) Effect of various factors on pull out strength of pedicle screw in normal and osteoporotic cancellous bone models. Medical engineering physics.40:28-38.

49. Meyer DC, Stalder M, Koch PP, Snedeker JG , Farshad M (2012) Contact pressure on ACL hamstring grafts in the bone tunnel with interference screw fixation-dynamic adaptation under load. The Knee.19:676-9. 